\title{
Optical Absorption Spectra of Nanocrystal Gold Molecules
}

\author{
Marcos M. Alvarez, Joseph T. Khoury, T. Gregory Schaaff, Marat N. Shafigullin, \\ Igor Vezmar, and Robert L. Whetten*
}

Schools of Physics and Chemistry and Microelectronics Research Center, Georgia Institute of Technology, Atlanta, Georgia 30332-0430

Received: September 23, 1996; In Final Form: March 4, $1997^{\otimes}$

\begin{abstract}
The optical absorption spectra of a series of nanocrystal gold molecules-larger, crystalline Au clusters that are passivated by a compact monolayer of $n$-alkylthiol(ate) $s$-have been measured across the electronic range $(1.1-4.0 \mathrm{eV})$ in dilute solution at ordinary temperature. Each of the $\sim 20$ samples, ranging in effective core diameter from 1.4 to $3.2 \mathrm{~nm}$ ( $\sim 70$ to $\sim 800 \mathrm{Au}$ atoms), has been purified by fractional crystallization and has undergone a separate compositional and structural characterization by mass spectrometry and X-ray diffraction. With decreasing core mass (crystallite size) the spectra uniformly show a systematic evolution, specifically (i) a broadening of the so-called surface-plasmon band until it is essentially unidentifiable for crystallites of less than $2.0 \mathrm{~nm}$ effective diameter, (ii) the emergence of a distinct onset for strong absorption near the energy $(\sim 1.7 \mathrm{eV})$ of the interbandgap $(5 \mathrm{~d} \rightarrow 6 \mathrm{sp})$, and (iii) the appearance in the smallest crystallites of a weak steplike structure above this onset, which is interpreted as arising from a series of transitions from the continuum d-band to the discrete level structure of the conduction band just above the Fermi level. The classical electrodynamic (Mie) theory, based on bulk optical properties, can reproduce this spectral evolution-and thereby yield a consistent core-sizing-only by making a strong assumption about the surface chemical interaction. Quantitative agreement with the spectral line shape requires a size-dependent offset of the frequency-dependent dielectric function, which may be explained by a transition in electronic structure just below $2.0 \mathrm{~nm}(\sim 200$ atoms), as proposed earlier.
\end{abstract}

\section{Introduction}

Nanometer-scale metal particles exhibit optical properties of great aesthetic, technological, and intellectual value. These properties are conveniently elucidated through conventional optical spectroscopic methods. ${ }^{1}$ At a fundamental level, optical absorption spectra provide information on the electronic structure of small metallic particles. To understand how these and other properties evolve from an atomic to a macroscopic state continues to be the most emotive cry in research on metal clusters and small particles. ${ }^{2}$ On a more practical level, the unique optical properties of small metallic particles are exploited in the manufacturing of optical filters as labels for biomacromolecules, in reversible photosensitive monochromatic glasses, ${ }^{3}$ for intensity enhancement in Raman spectroscopy (SER effect), ${ }^{4}$ for optical switching based on their large, ultrafast nonlinear optical response,,$^{5}$ and for optical trapping (or "tweezers"), based on their high polarizability. ${ }^{6}$ For their beauty and resilience, colloidal gold suspensions have found numerous decorative applications, such as in purple of Cassius and in the ruby glasses dating to the Middle Ages. ${ }^{7}$ In fact, it was the color variation of colloidal gold with size that motivated Mie to apply the general theory of light extinction to small particles. ${ }^{8}$

The absorption spectra of many metallic nanoparticles are characterized by a strong broad absorption band that is absent in the bulk spectra. Classically, this giant dipole (or surfaceplasmon) band is ascribed to a collective oscillation of the conduction electrons in response to optical excitation. ${ }^{9}$ The presence of this band in the visible region of the spectrum is responsible for the striking colors of dilute colloidal solutions of noble, alkali, alkaline earth $(\mathrm{Ca}, \mathrm{Sr}, \mathrm{Ba})$ and rare-earth (Eu, Yb) nanoparticles. ${ }^{10}$ Mie's theory predicts that below a certain size, less than one-tenth of the optical wavelength, the position and width of this band should remain constant, independent of

\footnotetext{
${ }^{\otimes}$ Abstract published in Advance ACS Abstracts, April 15, 1997.
}

size. ${ }^{7}$ Experimental evidence, however, indicates a slight but significant shift to lower energy accompanied by a dramatic increase in width with decreasing size. ${ }^{11}$ For free-electron metals, Fragstein and Kreibig and others ${ }^{12-14}$ advanced the theory that when the particle diameter becomes smaller than the electronic mean-free path in the bulk metal (ca. $20 \mathrm{~nm}$ for gold), the scattering of free electrons with the particle surface begins to affect their response to optical excitation. Such a simple and practical theory succeeds in explaining spectra of relatively large particles ( $>3 \mathrm{~nm}$ for gold) but stands on loose ground when applied to the smaller sizes. It is anticipated that at one point the phenomenological description of free electrons, as well as inherent fundamental assumptions of infinite lattice periodicity and a continuous energy-level spectrum, must fail. Thus far, however, it has not been possible to unequivocally identify quantum size effects in the optical spectra of metal nanoparticles prepared in macroscopic quantities, although such effects are well-known from experiments on metal-cluster beams $^{2}$ and from conductance measurements on single-metal nanostructures. ${ }^{15}$ One problem has been that experimental measurements for the small nanoparticles are often degraded by a lack of size and shape uniformity that renders comparison with theory questionable. ${ }^{16}$

This obstacle has been largely overcome by the discovery of methods for preparing gold:alkylthiolate assemblies that are the nanometer-scale analogue to the well-studied surface system. Specifically, on extended Au surfaces, one finds an extraordinary example ${ }^{17}$ of the uniform protection of a surface without modification of its essential structural and electronic properties. $n$-Alkylthiolates and their derivatives form compact, ordered monolayers in which thiolates $(\mathrm{RS}-$ ) or dialkyl disulfides (RSSR) reversibly attach to various $\mathrm{Au}$ surfaces. Their spontaneous assembly is driven by favorable (van der Waals) interactions among the long, ordered alkane chains in concert with a weak chemisorption of the sulfur head group to the metal 
surface $(\sim 1.2 \mathrm{eV}$ per RSSR unit desorbed versus $\sim 10 \mathrm{eV}$ surface energy per six Au atoms covered by this unit). These extensively studied systems have recently begun to be well understood. For example, a surface structure implicating interactions between $\mathrm{Au}$ surface atoms and the nonbonding $\mathrm{S}$ orbitals of an intact RSSR molecule has been identified. ${ }^{18}$

The subsequent discovery and increasingly wide exploration of the highly stable Au:SR nanocrystal systems over the past three years has established the stable existence and many properties of these systems; namely, they are charge-neutral entities comprised of a compact crystalline core of close-packed metal atoms and a dense mantle of straight-chain groups, which can be prepared quantitatively by at least two distinct methods ${ }^{19,20}$ effective across the $\sim 1.4-3.5 \mathrm{~nm}$ core diameter range ( 70-1000 atoms). Their surface properties are dictated by the surfactant tail-group (usually methyl, $-\mathrm{CH}_{3}$ ), resulting in weak interactions with external agents (e.g., solvents, strong acids, or bases) and with each other; the surfactant groups (RS) initially adsorbed can later be exchanged with excess thiol ( $\mathrm{R}^{\prime} \mathrm{SH}$ ) in solution. ${ }^{21-23}$ Their electronic properties are those characteristic of a small metal particle surrounded by a condensed dielectric medium. ${ }^{24}$ Most remarkably, the raw samples as prepared have been shown by mass spectrometry to contain enhanced abundances at certain sizes, presumably resulting from filled structural shells of $\mathrm{Au}$ atoms. The components of these mixtures can be fractionated from one another according to size and then handled as purified molecular substances in various ordered (thin films, molecular crystals) and dispersed phases. ${ }^{22,25}$ Considerable insight into the structure of these assemblies and their interactions has recently been provided by realistic simulations ${ }^{26}$ and by X-ray diffraction patterns obtained on selected samples that are matched to patterns calculated from theoretically generated structures. ${ }^{27} \mathrm{We}$ have recently ${ }^{28}$ found methods to produce and isolate smaller nanocrystals $(1.4-1.7 \mathrm{~nm})$ in large quantities, enabling one to obtain a rather complete picture of the evolution of the optical properties of gold.

\section{Experimental Methods and Results}

Examples of optical spectra obtained on samples of nanocrystal gold molecules are shown in Figures 1 and 2. These are purified fractions of materials prepared using hexyl- (denoted by $\left.\mathrm{SC}_{6}\right)$, dodecyl- $\left(\mathrm{SC}_{12}\right)$, and octadecyl- $\left(\mathrm{SC}_{18}\right)$ thiol(ate) surfactants and separated by methods described in refs 25 and 29 , and each has undergone a separate characterization involving mass spectrometry, large-angle X-ray diffraction (core structure), and small-angle X-ray diffraction on crystalline films or powders. Relevant mass-spectra are shown in Figure 3, and information extracted from XRD is shown in Figure 4. To obtain the optical spectra shown, toluene solutions of each fraction were prepared at dilutions such that the optical absorbance near $4 \mathrm{eV}$ was approximately unity. In practice, this involved a greater than 1000-fold dilution from the saturation limit. The absorption spectrum of each fraction was recorded from 1100 to $290 \mathrm{~nm}$ at normal temperatures using a Beckman DU 650 single-beam spectrometer $\left(A_{290} \approx 1\right.$ through a $1 \mathrm{~cm}$ quartz cell), and the solvent background is subtracted.

The spectra shown in Figure 1 are arbitrarily normalized at the high-energy end and are presented superimposed, without risk of confusion, because the relative spectral intensity at 2.5 $\mathrm{eV}$ decreases monotonically with mass (core size) for each series shown. Furthermore, Figure 2 illustrates that the spectra are largely independent of chain length for a given core mass, although at smaller sizes $(\sim 1.7 \mathrm{~nm})$ they can be quite sensitive to sample purity (Figure $2 b$ ).

The optical spectra are correlated with other characterization as follows. The mass spectral characterization shown in Figure

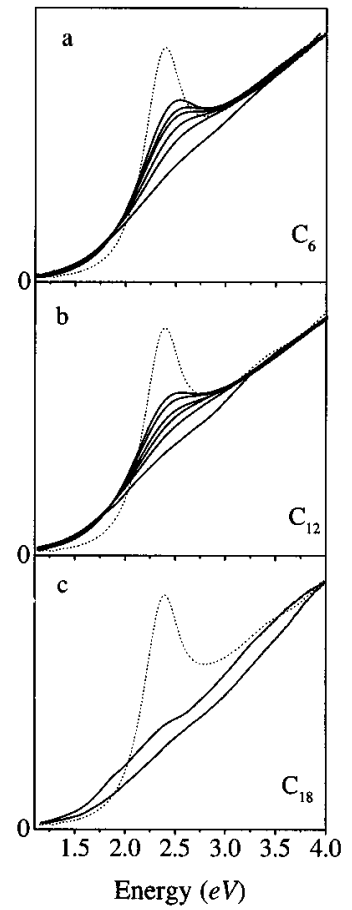

Figure 1. Optical absorption spectra (absorbance vs photon energy) for dilute solutions of several purified fractions of nanocrystal gold molecules passivated by (a) hexyl-, (b) dodecyl-, and (c) octadecylthiol(ates). The spectra are scaled to unity at $4 \mathrm{eV}$ and are compared to an aqueous solution of commercial colloidal Au particles (dotted lines) of $9 \mathrm{~nm}$ mean size. The peak amplitude (near $2.5 \mathrm{eV}$ ) descends with the metallic core diameters: (a) 3.2, 2.5, 2.4, 2.2, 2.0, and $1.7 \mathrm{~nm}\left(\mathrm{SC}_{6}\right.$ passivant); (b) 2.5, 2.4, 2.2, 2.1, 2.0, and $1.7 \mathrm{~nm}$ ( $\mathrm{SC}_{12}$ passivant); (c) 1.7 and $1.4 \mathrm{~nm}$ ( $\mathrm{SC}_{18}$ passivant).

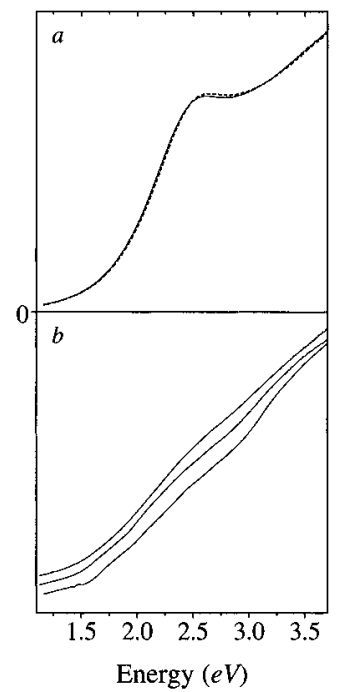

Figure 2. Optical spectra, as in Figure 1, comparing nanocrystals of like metallic cores. The upper frame contains spectra (normalized at 4 $\mathrm{eV}$ ) from $2.5 \mathrm{~nm}$ diameter clusters passivated with hexyl (solid line) and dodecyl (dashed). In the lower frame, several spectra of differently obtained $1.7 \mathrm{~nm}$ clusters are offset from each other: (top to bottom) passivated by $\mathrm{SC}_{6}, \mathrm{SC}_{12}$, and $\mathrm{SC}_{18}$.

3 has been used to extract a single quantity, the core mass, or approximate number of $\mathrm{Au}$ atoms, $N_{\mathrm{Au}}$, that can be converted into an effective core diameter $D_{\mathrm{MS}}$ using the density (59 atoms/ $\left.\mathrm{nm}^{3}\right)$ of bulk fcc-Au: $N_{\mathrm{Au}}=\left(59 \mathrm{~nm}^{-3}\right)(\pi / 6)\left(D_{\mathrm{MS}}\right)^{3}$. For convenience, this conversion is presented in graphical form in Figure $4 \mathrm{a}$. Figure $4 \mathrm{~b}$ demonstrates the correlation between the effective core diameter and the nearest-neighbor distance in the solid state, which is the most direct measure of the size of the overall assembly. The essence of the size-specific results shown in Figures 1-4, along with structural trends revealed by LA- 


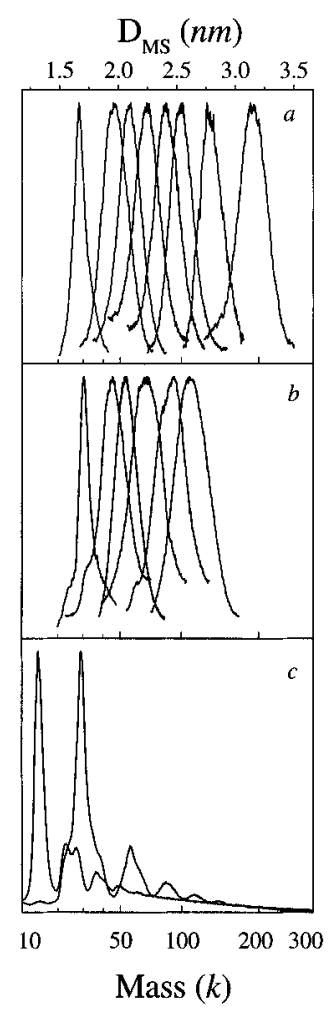

Figure 3. Sizing of the nanocrystal gold molecule fractions by mass spectrometry where the principal peaks from the mass spectra (compare to Figure 1) are displayed versus mass (in $k=1000$ amu units, lower axis, cube-root scale) and versus equivalent diameter ( $D_{\mathrm{Ms}}$, upper axis, linear scale): (a) 3.2, 2.7, 2.5, 2.4, 2.2, 2.1, 2.0, and $1.7 \mathrm{~nm}\left(\mathrm{SC}_{6}\right.$ passivant); (b) 2.5, 2.4, 2.2, 2.1, 2.0, and $1.7 \mathrm{~nm}$ (SC 12 passivant); (c) 1.7 and $1.4 \mathrm{~nm}$ ( $\mathrm{SC}_{18}$ passivant).

$\mathrm{XRD},{ }^{27}$ and the high crystallinity of condensed arrays formed from these samples ${ }^{31}$ are consistent with a uniformity of nanocrystallite structure that is seldom, if ever, attained in a series of macroscopically prepared materials.

Figures 5 and 6 present a diverse selection of the optical spectra replotted on a semilogarithmic scale (Figure 5) and in differential form (Figure 6). These bring out certain features to be discussed in the next section.

\section{Analysis and Discussion}

A. Qualitative Interpretation. In this section, we give a qualitative analysis and interpretation of the observed spectra, employing established facts concerning bulk fcc-Au, surfaces, and clusters. In the next section, the Mie theory and variants are applied in an attempt to gain a quantitative understanding of the spectra.

Superficially, the evolution of the optical spectrum of $\mathrm{Au}$ particles in the $1.4-3.2 \mathrm{~nm}$ size range $(\sim 70-800$ atoms) is a very simple one and could even be described as consistent with what is already known about Au from spectroscopic investigations of unfractionated samples of nanometer-scale particles in glasses, in solution, and from the smaller cluster compounds. ${ }^{32,33}$ Generally, these trends include the diminution, with decreasing cluster size, of the broad absorption band centered at $2.4 \mathrm{eV}$ until the spectrum is a nearly featureless straight line stretching from the near-infrared through the near-ultraviolet. However, the use of well-fractionated samples of gold nanocrystals, grown in a strongly etching environment, ${ }^{25}$ allows one to distinguish certain features that had not been noticed previously. These are the following.

(i) There is an onset to strong absorption located near 1.7 $\mathrm{eV}$ that becomes increasingly distinct at smaller nanocrystal sizes. (The same value can also be obtained, using the spectra
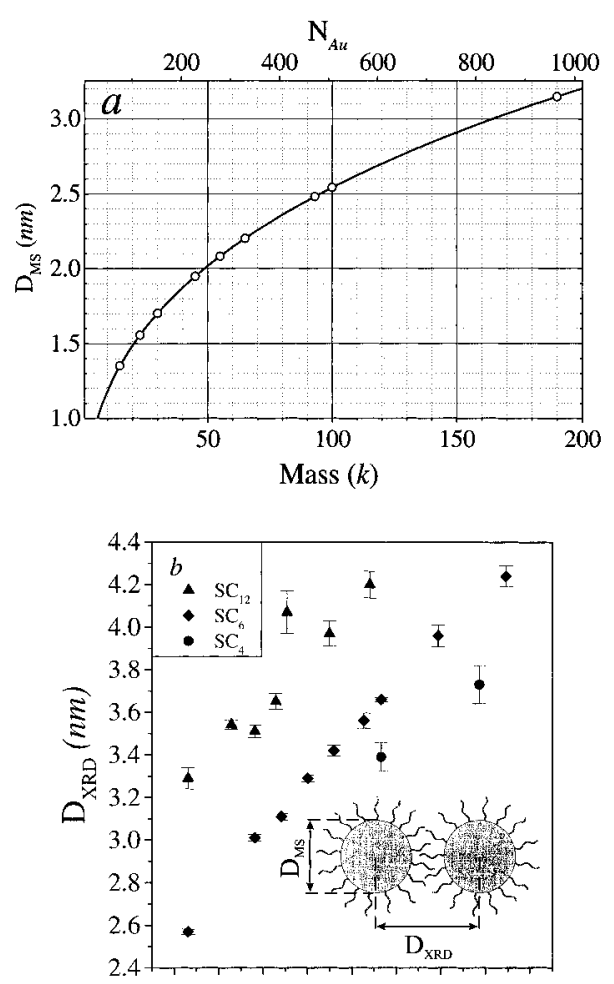

$\begin{array}{lllllllll}1.6 & 1.8 & 2.0 & 2.2 & 2.4 & 2.6 & 2.8 & 3.0 & 3.2\end{array}$

$$
\mathrm{D}_{\mathrm{MS}}(\mathrm{nm})
$$

Figure 4. (a) Conversion of the approximate number of Au atoms, $N_{\mathrm{Au}}$, into an effective core diameter $D_{\mathrm{Ms}}$ using the density (59 atoms/ $\left.\mathrm{nm}^{3}\right)$ of bulk fcc-Au: $N_{\mathrm{Au}}=\left(59 \mathrm{~nm}^{-3}\right)(\pi / 6)\left(D_{\mathrm{MS}}\right)^{3}$ presented in graphical form. (b) Correlation between the structural parameters, effective core diameter $\left(D_{\mathrm{MS}}\right)$ and nearest-neighbor center-to-center distance $\left(D_{\mathrm{XRD}}\right)$ for $(\boldsymbol{\Delta}) \mathrm{SC}_{12},(\bullet) \mathrm{SC}_{6}$, and $(\bullet) \mathrm{SC}_{4}$ passivated samples of gold nanocrystals.

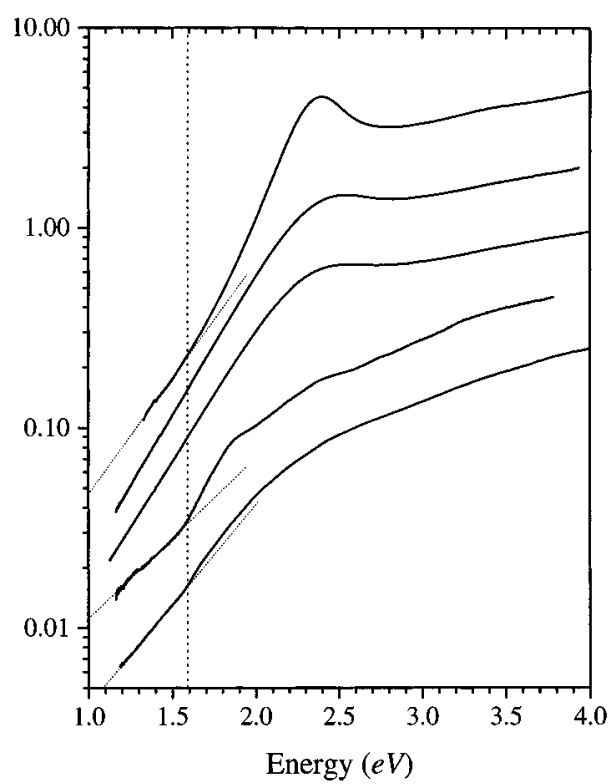

Figure 5. Optical spectra of diverse selected fractions replotted on a semilograthmic scale (offset for viewing): (from top to bottom) $9 \mathrm{~nm}$ colloidal gold and 3.2, 2.5, 1.7, and $1.4 \mathrm{~nm}$ nanocrystal gold molecules. The vertical dashed line marks the onset of the interband absorption just above $1.6 \mathrm{eV}$.

of larger-sized clusters, by extrapolating the linear region down to zero absorbance.) This can be seen even in the composite diagrams of Figures $1 \mathrm{c}$ and $2 \mathrm{~b}$ but is brought out particularly clearly by the spectra plotted semilogarithmically in Figure 5 . These show a clean break from the preonset behavior (dotted tangent lines) occurring at the energy $(\sim 1.6 \mathrm{eV}$, as marked by 


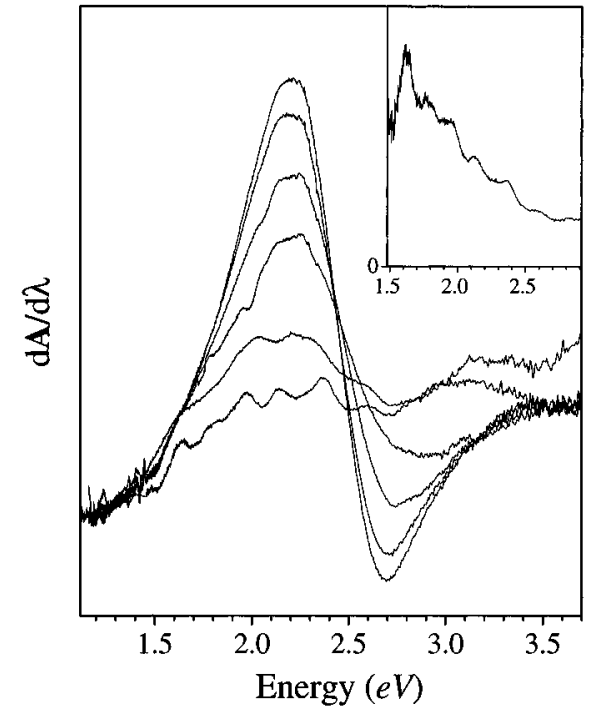

Figure 6. Derivative (with respect to wavelength $\lambda, \mathrm{d} A / \mathrm{d} \lambda$ ) of the optical absorption of diverse selected fractions: (from top to bottom) 2.6, 2.4, 2.2, 2.0, 1.7, and $1.4 \mathrm{~nm}$ diameter nanocrystal gold molecules. The inset shows the logarithmic derivative $\left(A^{-1} \mathrm{~d} A / \mathrm{d} \lambda\right)$ for the smallest of these. See text for discussion of the fine-structure appearing at smaller sizes.

the vertical dashed line) of the interband $(\mathrm{d} \rightarrow \mathrm{sp})$ absorption edge (see below).

(ii) A weak, steplike structure appears, starting from the onset just described, in the spectra of all the smallest clusters studied $(<2.0 \mathrm{~nm})$. Again, this can be seen even in the composite diagrams, particularly for the best-purified samples (lower curve of Figure 1c for $1.4 \mathrm{~nm}$ clusters and lowest curve of Figure $2 \mathrm{~b}$ for $1.7 \mathrm{~nm}$ clusters) but is most clearly evidenced in the differential (or log-differential) spectral intensities plotted in Figure 6 (and its inset), which transforms the weak steps into a series of distinct peaks and shoulders. These also bring out the onset structure $(1.7 \mathrm{eV})$ as a first peak.

The likely origin of these effects can be ascertained by considering the elementary facts of the electronic structure of close-packed $\mathrm{Au}$, as confirmed also to apply to Au clusters by cluster-beam experiments. ${ }^{34}$ Briefly, there are two sources of optical absorption intensity (in the $0-8 \mathrm{eV}$ range) in Au. Common to all metals are the intraband transitions occurring within the broad conduction band (derived mainly from Au $6 s^{1} \mathrm{p}$ hybridized atomic orbitals), which onset at zero frequency (or above the Kubo gap in small particles ${ }^{35}$ ). The resulting absorbance is quite weak, increasing as the square of the frequency until it approaches that of the "surface plasmon" frequency, which for conduction electron densities of $\mathrm{Au}(\sim 60$ / $\mathrm{nm}^{3}$ ) should appear near $5 \mathrm{eV}$ for the spherical geometry. In $\mathrm{Au}$, however, there are also the interband transitions occurring between the $5 \mathrm{~d}^{10}$ band and the unoccupied states of the conduction band, which have an onset at the energy difference between the highest point in the narrow, flat d-band and the lowest unoccupied level of the conduction band, i.e., at the Fermi level. In bulk $\mathrm{Au}$, this first interband excitation occurs at the $\mathrm{X}$-point of the first Brillouin zone, with an energy of $1.7 \mathrm{eV}$. The substantial $\mathrm{d} \rightarrow \mathrm{p}$ character of these transitions gives rise to very strong absorption and ultimately to the colors of $\mathrm{Au}$ surfaces, thin films, and nanoparticles. [The high polarizability of the $5 \mathrm{~d}^{10}$ cores (equivalent to a dielectric constant near 10) gives rise to a second effect, namely, a large red-shift of the collective-resonance to its observed location $(2.4 \mathrm{eV})$, with the unusual results that its intensity is dominated by the interband transitions, its existence by the intraband transitions, and its location by the latter but modified strongly by the polarizable $\mathrm{Au}^{+}$ion cores.] The breadth and proximity of this resonance in relation to the interband transition onset complicate identification of the interband absorption edge. However, the photoemission spectra of Taylor et al., ${ }^{34}$ obtained on massselected cold $\mathrm{Au}_{N}{ }^{-}$beams, show that this interband energy is well defined, at just below $2 \mathrm{eV}$, for clusters of $N=21$ to over 200 atoms, so that its location is probably constant for all closepacked Au clusters, regardless of the fraction of atoms at the surface.

Based on these facts, the onset feature observed near $1.7 \mathrm{eV}$ can be assigned without ambiguity to the first interband transitions, i.e., to transitions from the highest occupied dorbitals to the lowest unoccupied level(s) of the conduction band.

The interpretation of the discrete steplike structure (the peaks in Figure 6) is considerably less certain, since no such structure has previously been identified in condensed Au. By process of elimination, the evidence points toward the interpretation that it reflects the pattern of unoccupied energy levels located just above the Fermi level, i.e., from the quantum size effect in the conduction band. Each step would then represent a newly accessible channel in the sparse conduction band level structure for transitions from the quasicontinuum of d-band levels. [Similar arguments have been used for the spectra of insulator (e.g., CsI) and semiconductor $(\mathrm{CdS})$ clusters, where a very narrow valence band $\left(\mathrm{I}^{-} 5 \mathrm{p}^{6}\right.$ or $\left.\mathrm{S}^{2-} 3 \mathrm{p}^{6}\right)$ and broad conduction band $\left(\mathrm{Cs}^{+} 6 \mathrm{~s}\right.$ or $\left.\mathrm{Cd} 5 \mathrm{~s}\right)$ are involved. ${ }^{36,37}$ ] The argument supporting this conclusion rests on a detailed consideration of the density-of-states, degeneracies, and near-degeneracies (bunching) in various clusters in the $70-250$ atom size range and is beyond the scope of the present paper.

These considerations of the onset and steplike structure say nothing about the intensity profile of the above onset absorption and specifically the disappearance of the enhanced or collective effect, which are addressed by consideration of the Mie theory.

B. Application of the Mie Theory. In a quantitative analysis of the optical spectra of passivated gold nanocrystals, one can address the applicability of various size-dependent corrections to the optical properties derived from bulk Au. These include the effects of surface scattering (mean-free path correction), surfactant effects on the core electron density, and quantum size effects. In addition, one may obtain a quantitative way to provide size information (sizing) independent of mass, XRD, or TEM analysis. In the following, the basic Mie theory and size corrections are outlined and implemented by fitting to seven mass fractions passivated by $\mathrm{SC}_{12}$ surfactant. Only a brief description is presented here; a more complete description of this research can be found in ref 20 .

The main assumption of Mie's theory of the (linear) optical absorption by small particles is that the particle and its surrounding medium are each homogeneous and describable by their bulk optical dielectric functions. In general, solutions to Maxwell's equations for this geometry yield an expression for the absorption cross section, which is a sum over electric and magnetic multipoles (spherical vector harmonics and Legendre polynomials). When the size of a particle is much smaller than the wavelength of the exciting radiation, the absorption is dominated by the dipole term, with a cross section $\sigma(\omega)$ given by $^{7}$

$$
\sigma(\omega)=9 \epsilon_{\mathrm{m}}^{3 / 2} V_{0} \frac{\omega}{c} \frac{\epsilon_{2}(\omega)}{\left[\epsilon_{1}(\omega)+2 \epsilon_{\mathrm{m}}\right]^{2}+\epsilon_{2}{ }^{2}(\omega)}
$$

In this expression, $\omega$ is the frequency and $c$ the speed of light, $\epsilon_{\mathrm{m}}$ is the dielectric constant of the embedding medium (assumed frequency-independent over the spectral range of interest), $V_{0}$ is the volume of the absorbing particle, and $\epsilon_{1}(\omega)$ and $\epsilon_{2}(\omega)$ are the real and imaginary parts of the frequency-dependent 


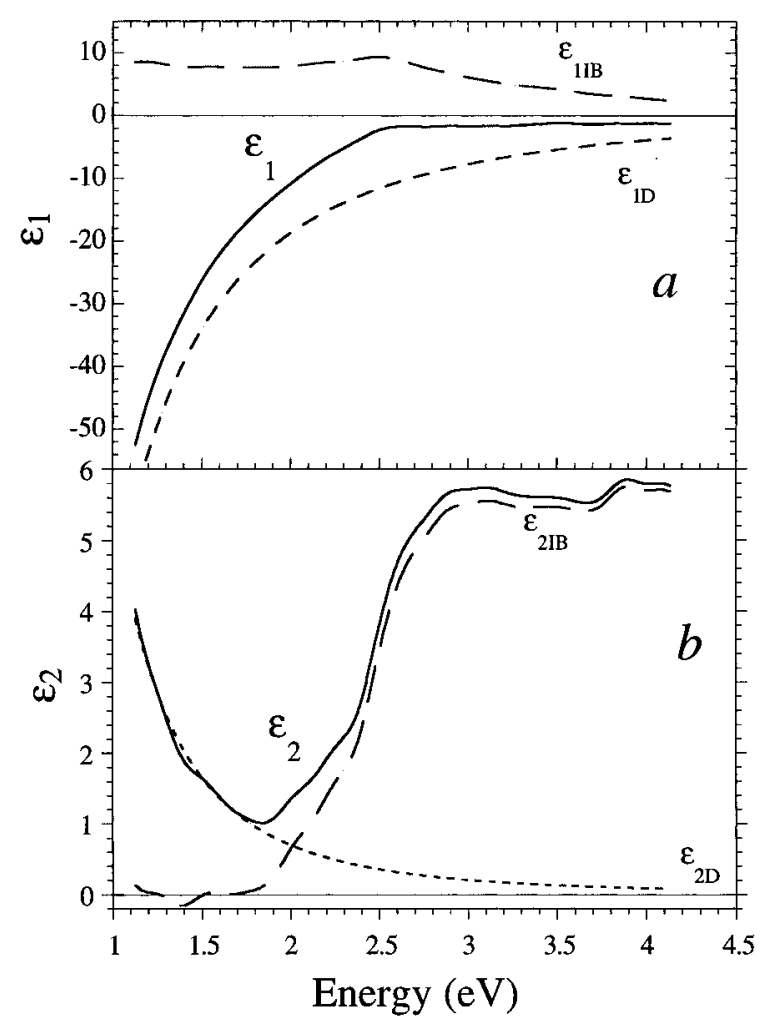

Figure 7. Decomposition of the experimental dielectric functions (solid lines) of bulk fcc-Au into free-electron (Drude) and interband ( $5 \mathrm{~d} \rightarrow$ 6sp) contributions: (a) real part; (b) imaginary part. The onset of significant interband absorption (dotted curve in lower frame) can be seen near $1.8 \mathrm{eV}$.

dielectric constant of the absorbing solid. [For a sample of dilute (noninteracting) absorbers of number density $n$ and path length $l_{0}, \sigma(\omega)$ is related to the conventional absorbance through the proportionality $A(\omega)=n \sigma(\omega) l_{0} / \ln (10)$.] The solid lines in Figures 7 and 8 give, respectively, the bulk dielectric function for $\mathrm{Au}$ and the corresponding absorption spectrum, calculated from eq 1 , based on the bulk optical constants ${ }^{39}$ extracted by a cubic spline fit. The spectrum (Figure 8), calculated assuming $\epsilon_{\mathrm{m}}=1$, shows a giant dipole resonance peaking near $2.3 \mathrm{eV}$, coinciding with the vanishing of $\epsilon_{1}(\omega)+2 \epsilon_{\mathrm{m}}$.

The spectral features predicted by eq 1 are size-independent, the particle dimension appearing only as a volumetric scaling factor. The first step in obtaining a size dependence for metal particles is the decomposition of the dielectric function into two terms: an interband contribution (IB), accounting for the response of 5d-electrons, and a free-electron contribution (Drude, $D)^{38}$ from the electrodynamics of the nearly free conduction electrons: ${ }^{12}$

$$
\epsilon_{1}(\omega)=\epsilon_{1 \mathrm{IB}}(\omega)+\epsilon_{1 \mathrm{D}}(\omega), \quad \epsilon_{2}(\omega)=\epsilon_{2 \mathrm{IB}}(\omega)+\epsilon_{2 \mathrm{D}}(\omega)
$$

where

$$
\epsilon_{1 \mathrm{D}}(\omega)=1-\frac{\omega_{\mathrm{p}}{ }^{2}}{\omega^{2}+\omega_{0}{ }^{2}}, \quad \epsilon_{2 \mathrm{D}}(\omega)=\frac{\omega_{\mathrm{p}}{ }^{2} \omega_{0}}{\omega\left(\omega^{2}+\omega_{0}{ }^{2}\right)}
$$

Here, $\omega_{\mathrm{p}}$ is the frequency of the plasma oscillation of free electrons expressed in terms of the free electron density $N$, the electron charge $e$, and effective mass $m$ :

$$
\omega_{\mathrm{p}}^{2}=\pi N e^{2} / m
$$

which corresponds energetically to $8.89 \mathrm{eV}$ for gold and 9.08 $\mathrm{eV}$ for silver. ${ }^{39}$ The term $\omega_{0}$, usually on the order of hundredths of an electronvolt, stands for the frequency of inelastic collisions

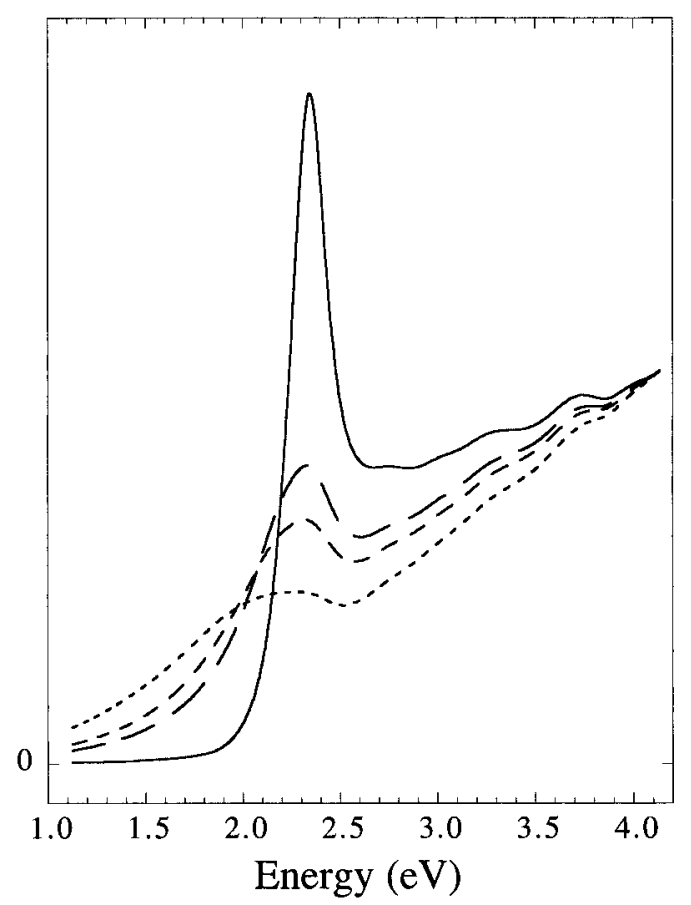

Figure 8. Absorption spectra predicted by Mie theory for particles of decreasing diameter: (from top to bottom) 40 (bulk), 3, 2, $1 \mathrm{~nm}$. All curves were normalized to unity at $4.1 \mathrm{eV}$. The size dependence results entirely from mean-free path corrections to both real and imaginary dielectric functions.

(electron-phonon coupling, defects, impurities) of free electrons within the metal. The separation of susceptibilities of bulk fcc$\mathrm{Au}$ is shown in Figure 7. The variation in the $\epsilon_{1}(\omega)$ is free electron in nature below $2.4 \mathrm{eV}$, although offset by the large, positive, and near-constant interband contribution. A significant inflection appears near $2.4 \mathrm{eV}$. The $\epsilon_{2}(\omega)$ function is dominated by the free electron term below $1.7 \mathrm{eV}$ and by the interband contribution above $2.4 \mathrm{eV}$.

To introduce size effects, one assumes that as the size of the particle diminishes, the rate of scattering from the particle surface $\left(\omega_{\mathrm{s}}\right)$ begins to greatly exceed the bulk scattering rate $\omega_{0} .{ }^{12}$ The surface scattering rate is expressed in terms of the Fermi velocity $\left(v_{\mathrm{F}}=1.4 \times 10^{8} \mathrm{~cm} / \mathrm{s}\right.$ for gold and silver $)$ and particle radius: ${ }^{12}$

$$
\omega_{\mathrm{s}}=A v_{\mathrm{F}} / R
$$

This expression can be interpreted as a limitation on the meanfree path of the free electrons by the particle dimensions. The proportionality factor, $A$, is of the order of unity and its meaning is not free of controversy. ${ }^{40}$ It takes the value unity if the scattering is assumed isotropic, 3/4 if diffusive, zero if elastic. Values from 0.1 to above 2 have been deduced and theoretically justified $^{11}$ as other factors are incorporated into the theory (electron density at the surface, effect of the interface, anisotropy of particle, quantum mechanical computations). To obtain dielectric functions of the particles, it is assumed that the interband contribution (Figure 7) is unchanged from the bulk but that free-electron contributions for small particles use $\omega_{\mathrm{s}}$ in place of $\omega_{0}$ in eq 3 .

The predicted size-evolution of the optical absorption spectrum obtained using this mean-free path correction $(A=1)$ is shown in Figure 8 for $\mathrm{Au}$ spheres in the size range $1-3 \mathrm{~nm}$ and is compared with the spectrum calculated assuming only the bulk scattering rate. The damping and red-shift of the giant dipole band are clearly evident. The red-shift in the position of the plasmon band arises from the correction to $\epsilon_{1}$, which is not negligible at smallest sizes. ${ }^{12}$ The rising slope of the 

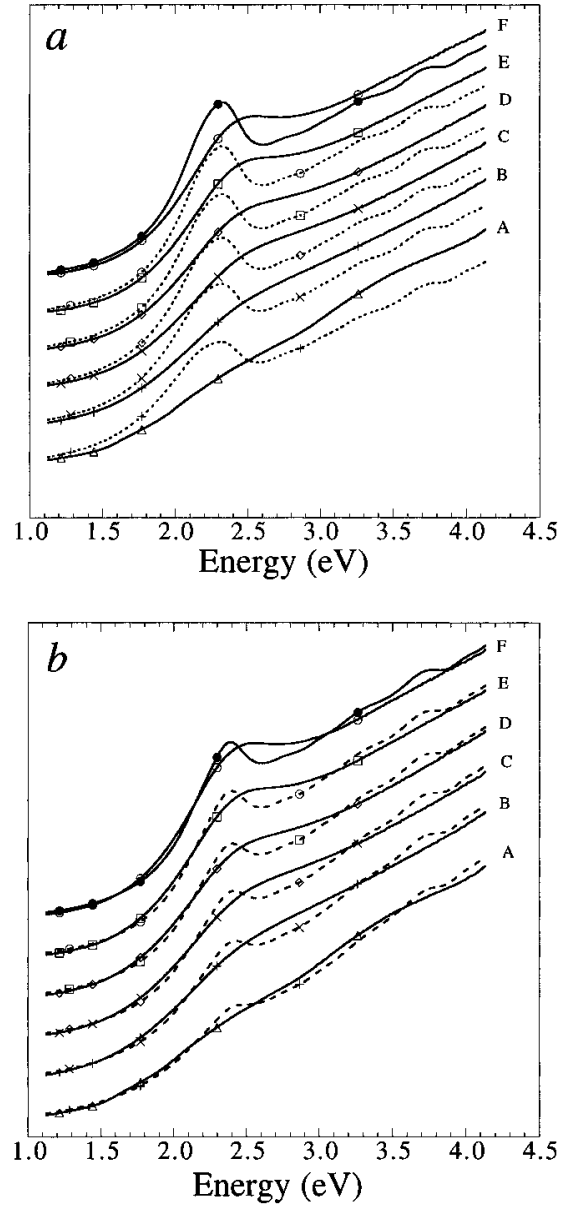

Figure 9. Comparison of experimental absorption spectra (solid lines) of seven $\mathrm{SC}_{12}$ gold fractions in hexane with predictions of the Mie theory (dotted lines). (a) The optical constants are modified by the meanfree path correction. Mass-derived diameters in nanometers are 1.7, $1.9,2.0,2.1,2.3$, and 2.5. All spectra are normalized to unity at 4.1 $\mathrm{eV}$ and displaced vertically. Optimal fits give extracted diameters, in nanometers, of 1.94, 2.2, 2.4, 2.58, 2.82, 3.02 for fractions $\mathrm{A}-\mathrm{F}$ respectively. (b) Optimal fits are shown incorporating the effect of electron donation from surfactant groups (dotted lines), as in (a). Optical constants were corrected with the mean-free path approximation. Extracted diameters are, in nanometers, 1.82, 1.74, 2.0, 2.22, 2.38, 2.64, and 2.84 for fractions $\mathrm{A}-\mathrm{G}$, respectively. Extracted $\mathrm{Au} / \mathrm{S}$ ratios are 4.0, 3.6, 3.3, 3.0, 2.8, and 2.1.

plasmon band decreases with decreasing size so that the onset of absorption is sharper for the largest particles.

In Figure 9, the predictions of the mean-free path correction are compared with the spectra obtained using $\mathrm{SC}_{12}$ surfactant groups (cf. Figure 1b), using the core radius as a fitting parameter (the extracted diameters are given in the caption). All curves have been arbitrarily normalized to unity at $4.1 \mathrm{eV}$ and displaced vertically for ease of comparison. Attempts to fit the measured absorption coefficients with Mie theory and a simple MFP correction (eq 5) to the Drude terms in the dielectric function are presented in Figure 9a. The fits are poor, failing to describe the broadening of the surface plasmons and their position. The functional behavior in the mean-free path model (Figure 8) demands a relative increase in low-energy absorption, accompanying the quenching of the surface plasmon, that is not observed.

A second correction takes into account that absorbates can significantly affect the metal's electronic properties, both for bulk surfaces and in small particles. ${ }^{41}$ The dependence in eq 4 of the plasmon frequency $\left(\omega_{\mathrm{p}}\right)$ on the free-electron density $N$ allows for a simple phenomenological description of the adsorbate effect. Since thiol(ate)s are intimately bound to the particle surface, it is possible that they provide or withdraw additional electron density at the interface. For self-assembled thiol monolayers on flat gold surfaces, one thiol occupies approximately $0.2 \mathrm{~nm}^{2}$ of surface, ${ }^{42}$ and the coverage density is increased in small particles because of curvature introduced by edges and vertices. ${ }^{26}$ We have implemented this correction, taking as adjustable parameters the number of electrons donated (or withdrawn), the core size, and thiol surface coverage density. An improved fit can be obtained only by assuming that the surfactant groups donate electrons. In fact, if one electron is donated per RS chain adsorbed, then the extracted core dimensions and surfactant packing densities take on reasonable values, and the fits shown in Figure $9 \mathrm{~b}$ are obtained. [To obtain these fits, the experimental absorption spectra for each mass fraction were fitted by those calculated using eq 1 , with the core diameter and electron density as parameters, using the general curve-fitting feature of a commercial graphics program (Kaleidagraph). The medium was assumed to be dodecylthiol with an index of refraction of 1.4 , and a thiol coverage density (electron donation density) of $0.25 / \mathrm{nm}^{2}$ of surface was assumed as an initial guess in the iterations.] The best-fit cluster diameters are consistently $10-15 \%$ larger than the values obtained from mass spectrometry. Optimal packing densities correspond to one RS group (one donated electron) per $4.0 \mathrm{Au}$ atoms at the surface of a $2.5 \mathrm{~nm}$ diameter particle and 1 per 2.1 $\mathrm{Au}$ surface atoms for a $1.8 \mathrm{~nm}$ radius particle, in reasonable agreement with recent realistic simulations of the structure of Au:alkylthiolate assemblies. ${ }^{26}$

These modifications fail to explain the abnormally wide or depressed collective oscillation band, which resists to be fitted with simple corrections even after introducing hypothetical cluster-size distributions, incorporating a Lorentz term for outer core electrons or invoking Kubo's quantum mechanical correction to the relaxation time. ${ }^{43}$ Physically, the passivating layer provides a dielectric coating on the surface of the sphere, amounting to a change with the dielectric constant of the medium. ${ }^{9,44}$ However, for the surfactants studied here, the effective index of refraction is that of the thiol layer (1.45 versus 1.37 for toluene). Chemical interface effects have sometimes been phenomenologically incorporated into the proportionality constant, $A$, to the surface scattering rate, ${ }^{40}$ but such a correction merely preserves the same functional form as the mean-free path correction. Another proposed modification does no better. Suppose that the outer surface gold atoms bound to the thiol(ate) lose their metallic character and are effectively removed from the metallic core, leaving its electron density unchanged. Such effect amounts to a reduction in the particle's radius by one gold lattice constant $(0.25 \mathrm{~nm})$, but sizes predicted by the theory are too large already.

Although lacking a specific theoretical foundation, it is possible mathematically to reproduce the spectra by modifying the dielectric functions, although this must be done in a way that satisfies certain constraints (sum rule and Kramers-Kronig relations). For example, a much improved fit is obtained simply by adding an energy-independent (offset) correction to the optical constants ${ }^{20}$ and suggests a transition in the electronic structure of Au at a size just below $2.0 \mathrm{~nm}$. Earlier, Kreibig ${ }^{45}$ has suggested that an abrupt change in the experimentally determined dielectric constants for small gold particles takes place at approximately $3 \mathrm{~nm}$ diameter. Our results place this change in the electronic structure of thiol-passivated gold particles precisely where a transition in the atom-packing structure has recently been found, ${ }^{27}$ suggesting that these may be interrelated.

We conclude that the optical spectra of passivated gold nanocrystals in the $1.4-3.2 \mathrm{~nm}$ diameter range cannot be explained in all detail with a mean-free path correction to gold's 
bulk dielectric constants. A simple model accounting for the effect of the passivating layer on the particle's electronic density, however, brings Mie theory into better agreement by assuming that thiolate adsorption increases the free-electron density of the particle in a proportion consistent with the manner in which alkane thiols are known to interact with bulk gold surfaces. The methodology employed here further allows the estimation of particle sizes within $20 \%$ accuracy. However, the breakdown of the model for the smallest sizes ( $1.4 \mathrm{~nm}$ diameter) and its failure to for the fine-structure (Figure 6) indicate that pronounced quantum size effects are operative. The implied corrections needed in the optical constants suggests the presence of a critical size around $2 \mathrm{~nm}$ for which the electronic structure changes dramatically.

\section{Concluding Remarks}

The evolution of the optical spectrum of passivated gold clusters in the $1.4-3.2 \mathrm{~nm}$ range ( $\sim 70$ to $\sim 900$ atoms) is a significant (strong) and systematic function of size. Because of this, the spectral profiles provide an independent, calibrated means of size determination useful for samples with narrow size distributions. Furthermore, the findings of an onset of interband absorption and the steplike structure beyond are indications that optical spectroscopy at high-frequency $(>1 \mathrm{eV}$ energies) may provide some precise information on the electronic structure of rather large metal clusters, a conclusion that runs against the conventional experience. ${ }^{1}$ Clearly, one requires measurements at cryogenic temperatures to remove thermal broadening effects, as well as continued efforts to improve the quality (molecular purity) and variety of materials investigated. The existence of these new materials of high stability and molecular quality suggests other spectroscopic avenues, including time-domain spectroscopy (see the accompanying article ${ }^{46}$ ) and measurements on thin crystalline films of condensed nanocrystal assemblies.

The quantitative analysis of the optical spectra of various samples of nanocrystal gold molecules, fractionated by size and separately characterized, confirms that their electronic and optical properties cannot be understood by any simple extrapolation from the bulk electronic properties of fcc-Au but rather require new concepts that may not yet have been clearly identified. Whether the evolution of the intensity profile and its substructure can yet be explained by a combination of quantum size effects (discrete level structure), changes in internal structure, and surface electronic characteristics remains to be seen.

Notes Added in Proof. Since this paper has been submitted, the smaller sizes (below $1.7 \mathrm{~nm}$ ) have been separated in sufficient quantities and their optical and electronic properties are discussed in a paper to be submitted.

Acknowledgment. This research has been supported by the Office of Naval Research, the Packard Foundation, and the Georgia Tech Research Research Foundation. The loan of the optical spectrometer by M. A. El-Sayed is gratefully acknowledged, as are helpful discussions with D. M. Chautz.

\section{References and Notes}

(1) Kreibig, U.; Vollmer, M. Optical Properties of Metal Clusters; Springer: Berlin, 1995.

(2) de Heer, W. A. Rev. Mod. Phys. 1993, 65, 612. Brack, M. Rev. Mod. Phys. 1993, 65, 677.

(3) Encyclopedia of Chemical Technology, 4th ed.; Wiley: New York, 1994; Vol. 12, pp 569-571.

(4) Freeman, R. G.; et al. Science 1995, 267, 1629.

(5) Fukumi, K.; Chayahara, A.; Kadono, K.; Sakaguchi, T.; Horino, Y.; Miya, M.; Fujii, K.; Hayakawa, J.; Satou, M. J. Appl. Phys. 1994, 75, 3075-80, and references therein.

(6) Svoboda, K.; Block, S. M. Opt. Lett. 1994, 19, 930-2.

(7) Read, for instance, the following. Ostwald, W. Theoretical and Applied Colloid Chemisty; Wiley: New, York, 1922; p 200. Zsigmondy,
R. The Chemisty of Colloids; Wiley: New York, 1917; p 156.

(8) Mie, G. Ann. Phys. 1908, 25, 377.

(9) Bohren, C. F.; Huffman, D. R. Absorption and Scattering of Light by Small Particles; Wiley: New York, 1983.

(10) Creighton, J. A.; Eadon, D. G. J. Chem. Soc., Faraday Trans. 1991, 87,3881 .

(11) Kreibig, U.; Genzel, L. Surf. Sci. 1985, 156, 678.

(12) Fragstein, C. V.; Kreibig, U. Z. Phys. 1969, 224, 306.

(13) Doremus, R. H. J. Chem. Phys. 1965, 42, 414.

(14) Doyle, W. T. Phys. Rev. 1958, B111, 1067.

(15) Ralph, D. C.; Black, C. T.; Tinkham, M. Phys. Rev. Lett. 1995 74, 3241-44. Black, C. T.; Ralph, D. C.; Tinkham, M. Phys. Rev. Lett. 1996, 76, 688-91.

(16) Huffman, D. R. In An Introduction to Physiscs and Technology of Thin Films; Wagendristerl, A., Wang, Y., Eds.; World Scientific: New Jersey, 1988.

(17) Dubois, L. H.; Nuzzo, R. G. Annu. Rev. Phys. Chem. 1992, 43, 437-463.

(18) Fenter, P.; Eberhardt, A.; Eisenberger, P. Science 1994, 266, 1216.

(19) Brust, M.; Walker, M.; Bethell, D.; Schiffrin, D. J.; Whyman, R. J. Chem. Soc., Chem. Commun. 1994, 801.

(20) Alvarez, M. M. Ph.D. Dissertation, UCLA, 1995. Alvarez, M. M.; Vezmar, I.; Whetten, R. L., submitted for publication. See also the following. Osifchin, R. G.; Mahoney, W. J.; Bielefeld, J. D.; Andres, R. P. Superlattices Microstruct., in press.

(21) Terrill, R. H.; Postlethwaite, T. A.; Chen, C.-H.; Poon, C.-D.; Terzis, A.; Chen, A.; Hutchison, J. E.; Clark, M. R.; Wignall, G.; Londono, J. D.; Superfine, R.; Falvo, M.; Johnson, C. S.; Samulski, E. T.; Murray, R. W. J. Am. Chem. Soc. 1995, 117, 12537.

(22) Whetten, R. L.; Khoury, J. T.; Alvarez, M. M.; Murthy, S.; Vezmar, I.; Wang, Z. L.; Cleveland, C. L.; Luedtke, W. D.; Landman, U. In Chemical Physics of Fullerenes 5 and 10 Years Later; Andreoni, W., Ed.; Kluwer: Dordrecht, 1996; pp 475-490. See also the following. Harfenist, S. A.; Wang, Z. L.; Alvarez, M. M.; Vezmar, I.; Whetten, R. L. J. Phys. Chem. 1996, 100, 13904-10.

(23) Badia, A.; Singh, S.; Demers, L.; Cuccia, L.; Brown, G. R.; Lennox, R. B. Chem.-Eur. J. 1996, 2, 205. Badia, A.; Gao, W.; Singh, S.; Demers, L.; Cuccia, L.; Reven, L. Langmuir 1996, 12, 1262-1269.

(24) Brust, M.; Bethell, D.; Schriffin, D. J.; Kiely, C. J. Adv. Mater. 1995, 7, 795-7.

(25) Whetten, R. L.; Khoury, J. T.; Alvarez, M. M.; Murthy, S.; Vezmar, I.; Wang, Z. L.; Stephens, P. W.; Cleveland, C. L.; Luedtke, W. D.; Landman, U. Adv. Mater. 1995, 8, 428-433.

(26) Luedtke, W. D.; Landman, U. J. Phys. Chem. 1996, 100, 1332329.

(27) Cleveland, C. L.; Landman, U.; Shafigullin, M. N.; Stephens, P. W.; Whetten, R. L. Structural evolution of larger gold clusters. Z. Phys. D, in press.

(28) Alvarez, M. M.; Khoury, J. T.; Schaaff, T. G.; Shafigullin, M. N.; Vezmar, I.; Whetten, R. L. Chem. Phys. Lett. 1997, 266, 91-8.

(29) Khoury, J. T.; et al. Rapid and quantitative sizing of passivated nanocrystals, submitted for publication.

(30) Vezmar, I.; Alvarez, M. M.; Khoury, J. T.; Salisbury, B. E.; Cluster beams from passivated nanocrystals. $Z$. Phys. $D$, in press.

(31) Shafigullin, M. N.; Alvarez, M. M.; Harfenist, S. A.; Khoury, J. T.; Schaaff, T. G.; Stephens, P. W.; Vezmar, I.; Wang, Z. L.; Wilkinson, A.; Whetten, R. L. The crystal structure of passivated-nanocrystal arrays, submitted for publication.

(32) Duff, D. G.; Baiker, A.; Edwards, P. P. J. Chem. Soc., Chem. Commun. 1993, 96-8.

(33) Fauth, K.; Kreibig, U.; Schmid, G. Z. Phys. D 1991, 20, 297.

(34) Taylor, K. J.; Pettiette-Hall, C. L.; Cheshnovsky, O.; Smalley, R. E. J. Chem. Phys. 1992, 96, 3319.

(35) Devaty, R. P.; Sievers, A. J. Phys. Rev. 1985, B32, 1951.

(36) Li, X.; Whetten, R. L. J. Chem. Phys. 1993, 98, 6170.

(37) For review, see the following. Alivisatos, A. P. J. Phys. Chem. 1996, 100,13226

(38) Ziman, J. M. Theory of Solids; University Press: Camobridge, 1979; p 278.

(39) Johnson, P. B.; Christy, R. W. Phys. Rev. 1972, B6, 4370-79.

(40) Hovel, H. S.; Fritz, A. H.; Kreibig, U.; Vollmer, M. Phys. Rev. $1993, B 48,18178$.

(41) Henglein, A. J. Phys. Chem. 1993, 97, 5457.

(42) Camillone, N., III; Chidsey, C. E. D.; Liu, G.; Scoles, G. J. Chem. Phys. 1993, 98, 4234.

(43) Kawabata, A.; Kubo, R. J. Phys. Soc. Jpn. 1966, 21, 1765.

(44) Linnert, T.; Mulvaney, P.; Henglein, A. J. Phys. Chem. 1993, 97, 679.

(45) Kreibig, U.; Hilger, A.; Hövel, H.; Quiten, M. In Large clusters of atoms and molecules; Martin, T. P., Ed.; Kluwer: Dordrecht, 1996; pp 4779 .

(46) Logunov, S.; Ahmadi, T. S.; El-Sayed, M. A.; Khoury, J. T.; Whetten, R. L. Electron dynamics of passivated gold nanocrystals probed by subpicosecond transient absorption spectroscopy. J. Phys. Chem. 1997, $101,3713$. 